\title{
SOME RESULTS OF OPERATOR IDEALS ON $s$-TYPE $|A, p|$ OPERATORS
}

\author{
AMIT MAJI AND P. D. SRIVASTAVA
}

\begin{abstract}
Let $s=\left(s_{n}\right)$ be a sequence of $s$-numbers in the sense of Pietsch and $A$ be an infinite matrix. This paper presents a generalized class $\mathscr{A}^{(s)}-p$ of $s$-type $|A, p|$ operators using $s$-number sequence which unifies many earlier well known classes. It is shown that the class $\mathscr{A}^{(s)}-p$ forms a quasi-Banach operator ideal under certain conditions on the matrix A. Moreover, the inclusion relations among the operator ideals as well as the inclusion relations among their duals are established. It is also proved that for the Cesàro matrix of order 1 , the operator ideal formed by approximation numbers is small for $1<$ $p<\infty$.
\end{abstract}

\section{Introduction}

Due to the immense applications in spectral theory, the geometry of Banach spaces, theory of eigenvalue distributions, etc., the theory of operator ideals occupies a special importance in functional analysis. Many useful operator ideals have been defined by using sequence of $s$-numbers. In 1963, Pietsch [4] firstly introduced the approximation numbers of a bounded linear operator in Banach spaces. Subsequently, different $s$-numbers, namely Kolmogorov numbers, Gel'fand numbers, etc. are introduced to the Banach space setting. For the unifications of different $s$-numbers, Pietsch $([5], 1974)$ defined an axiomatic theory of $s$ numbers in Banach spaces.

For each fixed infinite matrix $A=\left(a_{n k}\right)$, Rhoades [11] defined $A-p$ space, denoted by $|A, p|$ as

$$
|A, p|= \begin{cases}x \in w:\left(\sum_{n=1}^{\infty}\left(\sum_{k=1}^{\infty}\left|a_{n k} x_{k}\right|\right)^{p}\right)^{\frac{1}{p}}<\infty & \text { for } 0<p<\infty \\ x \in w: \sup _{n \geq 1}\left(\sum_{k=1}^{\infty}\left|a_{n k} x_{k}\right|\right)<\infty & \text { for } p=\infty,\end{cases}
$$

where $w$ is a sequence space of real or complex numbers. Further, Rhoades [12] has shown that if $A=\left(a_{n k}\right)$ is a triangle, i.e., $a_{n k}=0$ for $k>n$ and $a_{n n} \neq 0$, then the space $|A, p|$ is separable for $1<p<\infty$ and complete for $1<p \leq \infty$. $A-p$ spaces contain many known sequence

Received October 30, 2012, accepted February 18, 2014.

2010 Mathematics Subject Classification. 47B06, 47L20.

Key words and phrases. Approximation numbers, Kolmogorov numbers, operator ideals, $s$-numbers. Corresponding author: P. D. Srivastava. 
spaces such as Cesàro sequence spaces, $1 \leq p<\infty$ [13], $l_{p}$ sequence spaces, $0<p \leq \infty$ etc. by specifying suitable matrix. In particular, if we choose the matrix $A=\left(a_{n k}\right)$ as a Nörlund matrix, i.e.,

$$
a_{n k}= \begin{cases}\frac{a_{n+1-k}}{A_{n}}: & 1 \leq k \leq n \\ 0: & k>n,\end{cases}
$$

where $a_{n}$ is nonnegative for each $n$ and $A_{n}=\sum_{k=1}^{n} a_{k}>0$, then $|A, p|$ space reduces to

$$
\left\{x \in w:\left(\sum_{n=1}^{\infty}\left(\frac{1}{A_{n}} \sum_{k=1}^{n}\left|a_{n+1-k} x_{k}\right|\right)^{p}\right)^{\frac{1}{p}}<\infty\right\}
$$

whereas Nörlund sequence space [14] is defined as, for $1 \leq p<\infty$

$$
\left\{x \in w:\left(\sum_{n=1}^{\infty}\left(\frac{1}{A_{n}}\left|\sum_{k=1}^{n} a_{n+1-k} x_{k}\right|\right)^{p}\right)^{\frac{1}{p}}<\infty\right\} .
$$

If we choose the sequence $a_{n}=1$ for all $n \in \mathbb{N}$, then $|A, p|$ space reduces to Cesàro sequence space for $1 \leq p<\infty$, denoted as $c e s_{p}$. For $A=I$, we have the sequence space $l_{p}$ for $0<p \leq \infty$.

Pietsch [4] calls an operator $T \in \mathscr{L}(E, F)$ to be $l^{p}$ type if $\sum_{n=1}^{\infty}\left(a_{n}(T)\right)^{p}$ is finite for $0<p<$ $\infty$, where $\left(a_{n}(T)\right)$ is the sequence of approximation numbers of the bounded linear operator $T$. Later on Constantin [2] generalized the class of $l_{p}$ type operators to the class of $c e s-p$ type operators by using the Cesàro sequence spaces, where an operator $T \in \mathscr{L}(E, F)$ is called ces - $p$ type if $\sum_{n=1}^{\infty}\left(\frac{1}{n} \sum_{k=1}^{n} a_{k}(T)\right)^{p}$ is finite, $1<p<\infty$. Rhoades [11] further generalized the class of ces $-p$ type operators to the class of $A-p$ type operators, where $A=\left(a_{n k}\right)$ is an arbitrary infinite matrix. An operator $T \in \mathscr{L}(E, F)$ is said to be $A-p$ type operator if $\left(a_{n}(T)\right)$ is an element of the corresponding $|A, p|$ space, $0<p \leq \infty$. Pietsch [7] studied extensively operator ideals generated from the different $s$-number sequences by using Lorentz sequence spaces.

The purpose of this paper is to study a generalized class of operators using the sequence of $s$-numbers. We have also shown that the class $\mathscr{A}^{(s)}-p$ of $s$-type $|A, p|$ operators is a quasiBanach operator ideal under some certain conditions on the infinite matrix $A$, which is more general than the usual classes of operator ideals. Moreover, we have obtained various inclusion relations among the operator ideals as well as the inclusion relations among their duals. Finally, it is shown that for the Cesàro matrix of order 1 , the operator ideal formed by approximation numbers is small for $1<p<\infty$.

\section{Preliminaries}

Throughout this paper we denote $E, F$ as the real or complex Banach spaces and $\mathscr{L}(E, F)$ as the space of all bounded linear operators from $E$ to $F$. Let $\mathscr{L}$ be the class of all bounded 
linear operators between arbitrary Banach spaces. We denote $E^{\prime}$ as the dual of $E$ and $x^{\prime}$ is the continuous linear functional on $E . \mathbb{N}$ and $\mathbb{R}^{+}$stand for the set of all natural numbers and the set of all nonnegative real numbers, respectively. Let $x^{\prime} \in E^{\prime}$ and $y \in F$, then the map $x^{\prime} \otimes y: E \rightarrow F$ is defined by $\left(x^{\prime} \otimes y\right)(x)=x^{\prime}(x) y, x \in E$.

We now state few results which will be used in the sequel. Before it, we recall some basic definitions and terminologies of $s$-numbers of operators and operator ideals.

Definition 2.1. A finite rank operator is a bounded linear operator whose dimension of the range space is finite.

Definition 2.2 ([1], [9]). A map $s=\left(s_{n}\right): \mathscr{L} \rightarrow \mathbb{R}^{+}$assigning to every operator $T \in \mathscr{L}$ a nonnegative scalar sequence $\left(s_{n}(T)\right)_{n \in \mathbb{N}}$ is called an $s$-number sequence if the following conditions are satisfied:

(S1) monotonicity: $\|T\|=s_{1}(T) \geq s_{2}(T) \geq \cdots \geq 0, \quad$ for $T \in \mathscr{L}(E, F)$

(S2) additivity: $s_{m+n-1}(S+T) \leq s_{m}(S)+s_{n}(T), \quad$ for $S, T \in \mathscr{L}(E, F), m, n \in \mathbb{N}$

(S3) ideal property: $s_{n}(R S T) \leq\|R\| s_{n}(S)\|T\|, \quad$ for some $R \in \mathscr{L}\left(F, F_{0}\right), S \in \mathscr{L}(E, F)$ and $T \in$ $\mathscr{L}\left(E_{0}, E\right)$, where $E_{0}, F_{0}$ are arbitrary Banach spaces

(S4) rank property: If $\operatorname{rank}(T) \leq n$ then $s_{n}(T)=0$

(S5) norming property: $s_{n}\left(I: l_{2}^{n} \rightarrow l_{2}^{n}\right)=1$, where $I$ denotes the identity operator on the $n$ dimensional Hilbert space $l_{2}^{n}$.

We call $s_{n}(T)$ the $n$-th $s$-number of the operator $T$. For results on $s$-number sequence, refer ([1], [5], [7], [8], [9]).

We give some examples of $s$-number sequences of a bounded linear operator.

Let $T \in \mathscr{L}(E, F)$ and $n \in \mathbb{N}$.

The $n$-th approximation number, denoted by $a_{n}(T)$, is defined as

$$
a_{n}(T)=\inf \{\|T-L\|: \quad L \in \mathscr{L}(E, F), \operatorname{rank}(\mathrm{L})<\mathrm{n}\} .
$$

The $n$-th Gel'fand number, denoted by $c_{n}(T)$, is defined as

$$
c_{n}(T)=\inf \left\{\left\|T J_{M}\right\|: \quad M \subset E, \operatorname{codim}(\mathrm{M})<\mathrm{n}\right\},
$$

where $J_{M}: M \rightarrow E$ be the natural embedding from subspace $M$ of $E$ into $E$.

The $n$-th Kolmogorov number, denoted by $d_{n}(T)$, is defined as

$$
d_{n}(T)=\inf \left\{\left\|Q_{N}(T)\right\|: \quad N \subset F, \operatorname{dim}(\mathrm{N})<\mathrm{n}\right\},
$$


where $Q_{N}: E \rightarrow E / N$ be the quotient map from $E$ onto $E / N$.

The $n$-th Weyl number, denoted by $x_{n}(T)$, is defined as

$$
x_{n}(T)=\inf \left\{a_{n}(T A):\left\|A: l_{2} \rightarrow E\right\| \leq 1\right\},
$$

where $a_{n}(T A)$ is an $n$-th approximation number of the operator $T A$.

The $n$-th Chang number, denoted by $y_{n}(T)$, is defined as

$$
y_{n}(T)=\inf \left\{a_{n}(B T):\left\|B: F \rightarrow l_{2}\right\| \leq 1\right\},
$$

where $a_{n}(B T)$ is an $n$-th approximation number of the operator $B T$. The $n$-th Hilbert number, denoted by $h_{n}(T)$, is defined as

$$
h_{n}(T)=\sup \left\{a_{n}(B T A):\left\|B: F \rightarrow l_{2}\right\| \leq 1,\left\|A: l_{2} \rightarrow E\right\| \leq 1\right\} .
$$

Remark 2.1 ([9]). Among all the $s$-number sequences defined above, it is easy to verify that the approximation number, $a_{n}(T)$ is the largest and the Hilbert number, $h_{n}(T)$ is the smallest $s$-number sequence, i.e., $h_{n}(T) \leq s_{n}(T) \leq a_{n}(T)$ for any bounded linear operator $T$. If $T$ is compact and defined on a Hilbert space, then all the $s$-numbers coincide with the singular values of $T$, i.e., the eigenvalues of $|T|$, where $|T|=\left(T^{*} T\right)^{\frac{1}{2}}$.

Proposition 2.1 ([9], p.115). Let $T \in \mathscr{L}(E, F)$. Then $h_{n}(T) \leq x_{n}(T) \leq c_{n}(T) \leq a_{n}(T) \quad$ and $\quad h_{n}(T) \leq y_{n}(T) \leq d_{n}(T) \leq a_{n}(T)$.

Definition 2.3. ([9], p.90) An $s$-number sequence $s=\left(s_{n}\right)$ is called injective if, given any metric injection $J \in \mathscr{L}\left(F, F_{0}\right), s_{n}(T)=s_{n}(J T)$ for all $T \in \mathscr{L}(E, F)$.

Definition 2.4. ([9], p.95) An $s$-number sequence $s=\left(s_{n}\right)$ is called surjective if, given any metric surjection $Q \in \mathscr{L}\left(E_{0}, E\right), s_{n}(T)=s_{n}(T Q)$ for all $T \in \mathscr{L}(E, F)$.

Proposition 2.2. ([9], pp.90-94) The Gel'fand numbers and the Weyl numbers are injective.

Proposition 2.3. ([9], p.95) The Kolmogorov numbers and the Chang numbers are surjective.

The following lemma is required to prove our theorems.

Lemma $2.1([5]) .\left|s_{n}(T)-s_{n}(S)\right| \leq\|T-S\| \quad$ for $S, T \in \mathscr{L}(E, F)$ and $n=1,2, \cdots$.

Definition 2.5 ((Dual $s$-numbers) [5]). For each $s$-number sequence $s=\left(s_{n}\right)$, a dual $s$-number function $s^{D}=\left(s_{n}^{D}\right)$ is defined by

$$
s_{n}^{D}(T)=s_{n}\left(T^{\prime}\right) \quad \text { for all } T \in \mathscr{L},
$$

where $T^{\prime}$ is the dual of $T$. 
Definition 2.6 ([7], p.152)). An $s$-number sequence is called symmetric if $s_{n}(T) \geq s_{n}\left(T^{\prime}\right)$ for all $T \in \mathscr{L}$. If $s_{n}(T)=s_{n}\left(T^{\prime}\right)$ then the $s$-number sequence is said to be completely symmetric.

Now we state some known results of dual of an $s$-number sequence.

Theorem 2.1. ([7], p.152) The approximation numbers are symmetric, i.e., $a_{n}\left(T^{\prime}\right) \leq a_{n}(T)$ for $T \in \mathscr{L}$.

Remark 2.2. $a_{n}\left(T^{\prime}\right)=a_{n}(T)$ for every compact operator $T$ ( refer, C. V. Hutton [3]).

Theorem 2.2 ([7], p.153). Let $T \in \mathscr{L}$. Then

$$
c_{n}(T)=d_{n}\left(T^{\prime}\right) \quad \text { and } \quad c_{n}\left(T^{\prime}\right) \leq d_{n}(T)
$$

In addition, if $T$ is a compact operator then $c_{n}\left(T^{\prime}\right)=d_{n}(T)$.

Theorem 2.3. ([9], p.96) Let $T \in \mathscr{L}$. Then

$$
x_{n}(T)=y_{n}\left(T^{\prime}\right) \quad \text { and } \quad y_{n}(T)=x_{n}\left(T^{\prime}\right)
$$

i.e., Weyl numbers and Chang numbers are dual to each other.

Theorem 2.4 ([7], p.153). The Hilbert numbers are completely symmetric, i.e., $h_{n}(T)=h_{n}\left(T^{\prime}\right)$ for all $T \in \mathscr{L}$.

Definition 2.7 ([7], [10]). Let $\mathscr{L}$ be the class of all bounded linear operators between arbitrary Banach spaces and $\mathscr{L}(E, F)$ be the set of all such operators from $E$ to $F$. A sub collection $\mathscr{M}$ of $\mathscr{L}$ is said to be an ideal if each component $\mathscr{M}(E, F)=\mathscr{M} \cap \mathscr{L}(E, F)$ satisfies the following conditions:

$(O I 1)$ if $x^{\prime} \in E^{\prime}, y \in F$ then $x^{\prime} \otimes y \in \mathscr{M}(E, F)$;

(OI2) if $S, T \in \mathscr{M}(E, F)$ then $S+T \in \mathscr{M}(E, F)$;

(OI3) if $S \in \mathscr{M}(E, F), T \in \mathscr{L}\left(E_{0}, E\right)$ and $R \in \mathscr{L}\left(F, F_{0}\right)$ then $R S T \in \mathscr{M}\left(E_{0}, F_{0}\right)$.

Definition 2.8 ([7], [10]). A function $\alpha: \mathscr{M} \rightarrow \mathbb{R}^{+}$is said to be a quasi-norm on the ideal $\mathscr{M}$ if the following conditions hold:

$(Q O N 1)$ if $x^{\prime} \in E^{\prime}, y \in F$ then $\alpha\left(x^{\prime} \otimes y\right)=\left\|x^{\prime}\right\|\|y\|$;

(QON2) there exists a constant $C_{\alpha} \geq 1$ such that $\alpha(S+T) \leq C_{\alpha}[\alpha(S)+\alpha(T)]$ for $S, T \in \mathscr{M}(E, F)$;

$(Q O N 3)$ if $S \in \mathscr{M}(E, F), T \in \mathscr{L}\left(E_{0}, E\right)$ and $R \in \mathscr{L}\left(F, F_{0}\right)$ then $\alpha(R S T) \leq\|R\| \alpha(S)\|T\|$. 
In particular if $C_{\alpha}=1$ then $\alpha$ becomes a norm on the operator ideal $\mathscr{M}$.

An ideal $\mathscr{M}$ with a quasi-norm $\alpha$, denoted by $[\mathscr{M}, \alpha]$ is said to be a quasi-Banach operator ideal if each component $\mathscr{M}(E, F)$ is complete under the quasi-norm $\alpha$. A quasi-normed operator ideal $[\mathscr{M}, \alpha]$ is called injective if for every operator $T \in \mathscr{L}(E, F)$ and a metric injection $J \in \mathscr{L}\left(F, F_{0}\right), J T \in \mathscr{M}\left(E, F_{0}\right)$ we have $T \in \mathscr{M}(E, F)$ and $\alpha(J T)=\alpha(T)$. Further, a quasi-normed operator ideal $[\mathscr{M}, \alpha]$ is called surjective if for every operator $T \in \mathscr{L}(E, F)$ and a metric surjection $Q \in \mathscr{L}\left(E_{0}, E\right), T Q \in \mathscr{M}\left(E_{0}, F\right)$ we have $T \in \mathscr{M}(E, F)$ and $\alpha(T Q)=\alpha(T)$. Thus injectivity and surjectivity are dual concept. For its various properties, please refer to [7].

Definition 2.9 ([7], [10]). For every operator ideal $\mathscr{M}$, the dual operator ideal denoted by $\mathscr{M}^{\prime}$ is defined as

$$
\mathscr{M}^{\prime}(E, F)=\left\{T \in \mathscr{L}(E, F): \quad T^{\prime} \in \mathscr{M}\left(F^{\prime}, E^{\prime}\right)\right\},
$$

where $T^{\prime}$ is the dual of $T$ and $E^{\prime}$ and $F^{\prime}$ are the duals of $E$ and $F$, respectively.

Definition 2.10 ([7], p.68). An operator ideal $\mathscr{M}$ is called symmetric if $\mathscr{M} \subset \mathscr{M}^{\prime}$ and is called completely symmetric if $\mathscr{M}=\mathscr{M}^{\prime}$.

Remark 2.3 ([7], [10]).

1. $\mathscr{M}^{\prime}$ is complete if $\mathscr{M}$ is complete.

2. If $\left[\mathscr{M}^{\prime}, \alpha^{\prime}\right]$ be the dual of quasi-normed ideal $[\mathscr{M}, \alpha]$ then $\alpha^{\prime}(T)=\alpha\left(T^{\prime}\right)$.

We now consider some known operator ideals determined by sequence of $s$-numbers, namely $\mathscr{L}_{r, p}^{(s)}$ and $S_{p}^{(s)}$ (see [7], [9]), where

$$
\mathscr{L}_{r, p}^{(s)}:=\left\{T \in \mathscr{L}: \sum_{n=1}^{\infty}\left(n^{\frac{1}{r}-\frac{1}{p}} s_{n}(T)\right)^{p}<\infty\right\} \text { for } 0<r, p<\infty
$$

and

$$
S_{p}^{(s)}:=\left\{T \in \mathscr{L}: \sum_{n=1}^{\infty}\left(s_{n}(T)\right)^{p}<\infty\right\} \quad \text { for } 0<p<\infty .
$$

Definition 2.11 ([6]). An operator ideal $\mathscr{M}$ is said to be small if $\mathscr{M}(E, F)=\mathscr{L}(E, F)$ implies that at least one of the Banach spaces $E$ and $F$ is of finite dimension.

\section{Operators of $s$-type $|A, p|$}

In this section we have defined $s$-type $|A, p|$ operators and proved that the operator ideal formed by $s$-number sequence is complete. We have also investigated the dual and inclusion results among the operator ideals. 
Let $s=\left(s_{n}\right)$ be a sequence of $s$-numbers. We call an operator $T \in \mathscr{L}(E, F)$ is of $s$-type $|A, p|$ operator if the sequence of $s$-numbers of $T,\left(s_{n}(T)\right)$ is an element of the corresponding $|A, p|$ space. In other words, $T$ is of $s$-type $|A, p|$ operator if

$$
\begin{cases}\left(\sum_{n=1}^{\infty}\left(\sum_{k=1}^{\infty}\left|a_{n k} s_{k}(T)\right|\right)^{p}\right)^{\frac{1}{p}}<\infty & \text { for } 0<p<\infty \\ \sup _{n \geq 1}\left(\sum_{k=1}^{\infty}\left|a_{n k} s_{k}(T)\right|\right)<\infty & \text { for } p=\infty\end{cases}
$$

For each fixed matrix $A$, we denote $\mathscr{A}^{(s)}-p$ be the class of all $s$-type $|A, p|$ operators between arbitrary Banach spaces and $\mathscr{A}_{(E \rightarrow F)}^{(s)}-p$ be the set of $s$-type $|A, p|$ operators from $E$ to $F$ which is a component of $\mathscr{A}^{(s)}-p$ for $0<p \leq \infty$.

Let $A=\left(a_{n k}\right)$ be a matrix satisfying the condition:

$$
\left|a_{n, 2 k-1}\right|+\left|a_{n, 2 k}\right| \leq M\left|a_{n k}\right| \quad \text { for each } k \text { and } n,
$$

where $M$ is a constant independent of $n$ and $k$.

Theorem 3.1. Let $0<p<\infty$. For fixed matrix $A=\left(a_{n k}\right)$ satisfying (3.1) and $\sum_{n=1}^{\infty}\left|a_{n 1}\right|^{p}<\infty$, the class $\mathscr{A}^{(s)}-p$ is an operator ideal.

Proof. Let $E$ and $F$ be any two Banach spaces. We shall prove $(O I 1)$ to $(O I 3)$ to show $\mathscr{A}^{(s)}-p$ is an operator ideal. Let $x^{\prime} \in E^{\prime}, y \in F$ then $x^{\prime} \otimes y$ is a rank one operator. So

$$
s_{n}\left(x^{\prime} \otimes y\right)=0 \quad \text { for all } n \geq 2 .
$$

We have

$$
\begin{aligned}
\left(\sum_{n=1}^{\infty}\left(\sum_{k=1}^{\infty}\left|a_{n k} s_{k}\left(x^{\prime} \otimes y\right)\right|\right)^{p}\right)^{\frac{1}{p}} & =\left(\sum_{n=1}^{\infty}\left(\left|a_{n 1} s_{1}\left(x^{\prime} \otimes y\right)\right|\right)^{p}\right)^{\frac{1}{p}} \\
& =\left\|x^{\prime} \otimes y\right\|\left(\sum_{n=1}^{\infty}\left|a_{n 1}\right|^{p}\right)^{\frac{1}{p}}<\infty .
\end{aligned}
$$

Thus $x^{\prime} \otimes y \in \mathscr{A}_{(E \rightarrow F)}^{(s)}-p$ and hence $(O I 1)$ is proved.

Let $S, T \in \mathscr{A}_{(E \rightarrow F)}^{(s)}-p$. We calculate

$$
\begin{aligned}
\sum_{k=1}^{\infty}\left|a_{n k} s_{k}(T+S)\right| & =\sum_{k=1}^{\infty}\left|a_{n, 2 k-1} s_{2 k-1}(T+S)\right|+\sum_{k=1}^{\infty}\left|a_{n, 2 k} s_{2 k}(T+S)\right| \\
& \leq\left(\sum_{k=1}^{\infty}\left(\left|a_{n, 2 k-1}\right|+\left|a_{n, 2 k}\right|\right) s_{2 k-1}(T+S)\right)
\end{aligned}
$$




$$
\leq M\left(\sum_{k=1}^{\infty}\left|a_{n k}\right| s_{k}(T)+\sum_{k=1}^{\infty}\left|a_{n k}\right| s_{k}(S)\right) .
$$

Case I: $0<p<1$

For $0<p<1$ and $a, b>0$, we have $(a+b)^{p} \leq\left(a^{p}+b^{p}\right)$ and $(a+b)^{\frac{1}{p}} \leq C\left(a^{\frac{1}{p}}+b^{\frac{1}{p}}\right)$, where $C \geq 1$ is a constant.

From (3.2), we have

$$
\begin{aligned}
\left(\sum_{n=1}^{\infty}\left(\sum_{k=1}^{\infty}\left|a_{n k} s_{k}(T+S)\right|\right)^{p}\right)^{\frac{1}{p}} & \leq M\left(\sum_{n=1}^{\infty}\left(\sum_{k=1}^{\infty}\left|a_{n k}\right| s_{k}(T)+\sum_{k=1}^{\infty}\left|a_{n k}\right| s_{k}(S)\right)^{p}\right)^{\frac{1}{p}} \\
& \leq C \cdot M\left[\left(\sum_{n=1}^{\infty}\left(\sum_{k=1}^{\infty}\left|a_{n k}\right| s_{k}(T)\right)^{p}\right)^{\frac{1}{p}}+\left(\sum_{n=1}^{\infty}\left(\sum_{k=1}^{\infty}\left|a_{n k}\right| s_{k}(S)\right)^{p}\right)^{\frac{1}{p}}\right]<\infty
\end{aligned}
$$

where $C \geq 1$ is a constant.

Case II: $1 \leq p<\infty$

Using Minkowski inequality for $1 \leq p<\infty$, we have from (3.2)

$$
\begin{aligned}
\left(\sum_{n=1}^{\infty}\left(\sum_{k=1}^{\infty}\left|a_{n k} s_{k}(T+S)\right|\right)^{p}\right)^{\frac{1}{p}} & \leq M\left(\sum_{n=1}^{\infty}\left(\sum_{k=1}^{\infty}\left|a_{n k}\right| s_{k}(T)+\sum_{k=1}^{\infty}\left|a_{n k}\right| s_{k}(S)\right)^{p}\right)^{\frac{1}{p}} \\
& \leq M\left[\left(\sum_{n=1}^{\infty}\left(\sum_{k=1}^{\infty}\left|a_{n k}\right| s_{k}(T)\right)^{p}\right)^{\frac{1}{p}}+\left(\sum_{n=1}^{\infty}\left(\sum_{k=1}^{\infty}\left|a_{n k}\right| s_{k}(S)\right)^{p}\right)^{\frac{1}{p}}\right]<\infty
\end{aligned}
$$

Thus $S+T \in \mathscr{A}_{(E \rightarrow F)}^{(s)}-p$ and hence (OI2) is proved.

Let $T \in \mathscr{L}\left(E_{0}, E\right), R \in \mathscr{L}\left(F, F_{0}\right)$ and $S \in \mathscr{A}_{(E \rightarrow F)}^{(s)}-p$. It is required to prove $R S T \in \mathscr{A}_{\left(E_{0} \rightarrow F_{0}\right)}^{(s)}-p$. Using the property (S3) in the Definition 2.2., we have

$$
s_{n}(R S T) \leq\|R\| s_{n}(S)\|T\| \quad \text { for all } n \in \mathbb{N} .
$$

So

$$
\begin{aligned}
\left(\sum_{n=1}^{\infty}\left(\sum_{k=1}^{\infty}\left|a_{n k} s_{k}(R S T)\right|\right)^{p}\right)^{\frac{1}{p}} & \leq\|R\|\|T\|\left(\sum_{n=1}^{\infty}\left(\sum_{k=1}^{\infty}\left|a_{n k} s_{k}(S)\right|\right)^{p}\right)^{\frac{1}{p}} \\
& <\infty
\end{aligned}
$$

Thus $R S T \in \mathscr{A}_{\left(E_{0} \rightarrow F_{0}\right)}^{(s)}-p$ and therefore (OI3) is proved.

Hence $\mathscr{A}^{(s)}-p$ is an operator ideal.

Corollary 3.1. Let $A=\left(a_{n k}\right)$ be a matrix satisfying (3.1) and $\sup \left|a_{n 1}\right|<\infty$. Then for $p=\infty$, the class $\mathscr{A}^{(s)}-\infty$ is an operator ideal. 
Remark 3.1. Let $A=\left(a_{n k}\right)$ be a matrix such that $\sum_{n=1}^{\infty}\left|a_{n 1}\right|^{p}<\infty$, then the condition (3.1) on the matrix is sufficient but not necessary to form an operator ideal. Justification is given below.

Justification: Let $0<p<\infty$ and $A=\left(a_{n k}\right)$ be a nonzero diagonal matrix such that

$$
\left|a_{2 n-1,2 n-1}\right|^{p}+\left|a_{2 n, 2 n}\right|^{p} \leq M_{1}\left|a_{n n}\right|^{p},
$$

where $M_{1}$ is a constant independent of $n$.

First of all, we show that for the nonzero diagonal matrix $A$ satisfying (3.3), the class $\mathscr{A}^{(s)}-p$ of $s$-type $|A, p|$ operators forms an operator ideal. For this let $S, T \in \mathscr{A}_{(E \rightarrow F)}^{(s)}-p$. Then

$$
\begin{aligned}
\left(\sum_{n=1}^{\infty}\left(\sum_{k=1}^{\infty}\left|a_{n k} s_{k}(T+S)\right|\right)^{p}\right)^{\frac{1}{p}} & =\left(\sum_{n=1}^{\infty}\left(\left|a_{n n}\right| s_{n}(T+S)\right)^{p}\right)^{\frac{1}{p}} \\
& \leq C_{1} \cdot M_{1}^{\frac{1}{p}}\left[\left(\sum_{n=1}^{\infty}\left(\left|a_{n n}\right| s_{n}(T)\right)^{p}\right)^{\frac{1}{p}}+\left(\sum_{n=1}^{\infty}\left(\left|a_{n n}\right| s_{n}(S)\right)^{p}\right)^{\frac{1}{p}}\right] \\
& =C_{1} \cdot M_{1}^{\frac{1}{p}}\left[\left(\sum_{n=1}^{\infty}\left(\sum_{k=1}^{\infty}\left|a_{n k} s_{k}(T)\right|\right)^{p}\right)^{\frac{1}{p}}+\left(\sum_{n=1}^{\infty}\left(\sum_{k=1}^{\infty}\left|a_{n k} s_{k}(S)\right|\right)^{p}\right)^{\frac{1}{p}}\right] \\
& <\infty .
\end{aligned}
$$

Thus $S+T \in \mathscr{A}_{(E \rightarrow F)}^{(s)}-p$. Hence (OI2) is proved. Clearly the conditions (OI1) and (OI3) hold good. Thus the class $\mathscr{A}^{(s)}-p$ is an operator ideal. But the nonzero diagonal matrix $A$ (In particular identity matrix) does not satisfy the condition (3.1). This proves our claim.

Remark 3.2. Let $A=I$, an identity matrix, then the operator ideal $\mathscr{A}^{(s)}-p$ becomes a well known operator ideal $S_{p}^{(s)}$ which has been studied extensively by many mathematicians. If we choose the matrix $A=\left(a_{n k}\right)$ such that for $0<r, p<\infty$

$$
a_{n k}= \begin{cases}n^{\frac{1}{r}-\frac{1}{p}}: & \text { if } n=k \\ 0: & \text { otherwise. }\end{cases}
$$

Then the matrix $A$ satisfies the condition (3.3) and forms a quasi-Banach operator ideals denoted as $\mathscr{L}_{r, p}^{(s)}$ introduced by Pietsch.

Note 3.1. It is observed that if the matrix $A=\left(a_{n k}\right)$ satisfies the condition (3.1), then the set $\mathscr{A}_{(E \rightarrow F)}^{(s)}-p$ of $s$-type $|A, p|$ operators from $E$ to $F$ is a linear space. So if we choose the $s$-number sequence as the sequence of approximation numbers, then the set $\mathscr{A}_{(E \rightarrow F)}^{(a)}-p$ is same as the set of $A-p$ type operators studied by Rhoades. If we choose the matrix $A$ as the Cesàro matrix of order 1 then the set $\mathscr{A}_{(E \rightarrow F)}^{(a)}-p$ coincides with the set of $c e s-p$ type operators introduced by Constantin. 
Note 3.2. Rhoades [11] raised an open question whether the condition (3.1) on the matrix $A=\left(a_{n k}\right)$ is necessary for the set of $A-p$ type operator to form a linear space? It is observed that the identity matrix $I$ does not satisfy the condition (3.1) but the set of $A-p$ type (here $A=I$ ) operators forms a linear space structure. This answers the question of Rhoades in negation, i.e., the condition (3.1) on the matrix $A$ is sufficient but not a necessary to form a linear space.

Proposition 3.1. For $1 \leq p<q \leq \infty$, we have $\mathscr{A}^{(s)}-p \subseteq \mathscr{A}^{(s)}-q$.

Proof. For $1 \leq p<q \leq \infty$, we have $|A, p| \subseteq|A, q|$. So the proof of Proposition 3.1. is trivial.

Let $\mathscr{A}^{(s)}-p$ be an operator ideal. Define $\beta_{A, p}^{(s)}: \mathscr{A}^{(s)}-p \rightarrow \mathbb{R}^{+}$for $0<p<\infty$ by

$$
\beta_{A, p}^{(s)}(T)=\left(\sum_{n=1}^{\infty}\left(\sum_{k=1}^{\infty}\left|a_{n k} s_{k}(T)\right|\right)^{p}\right)^{\frac{1}{p}}
$$

where $T \in \mathscr{A}^{(s)}-p$.

Note 3.3. For $p=\infty$, we define $\beta_{A, \infty}^{(s)}(T)=\sup _{n \geq 1}\left(\sum_{k=1}^{\infty}\left|a_{n k} s_{k}(T)\right|\right)$.

Theorem 3.2. Let $0<p<\infty$. For fixed nonzero matrix $A=\left(a_{n k}\right)$ satisfying the condition (3.1) and $\sum_{n=1}^{\infty}\left|a_{n 1}\right|^{p}<\infty$, the function $\hat{\beta}_{A, p}^{(s)}$ is a quasi-norm on the operator ideal $\mathscr{A}^{(s)}-p$, where $\hat{\beta}_{A, p}^{(s)}(T)=\frac{\beta_{A, p}^{(s)}(T)}{\left(\sum_{n=1}^{\infty}\left|a_{n 1}\right|^{p}\right)^{\frac{1}{p}}}, \quad T \in \mathscr{A}^{(s)}-p$.

Proof. Let $E$ and $F$ be two Banach spaces and $\mathscr{A}_{(E \rightarrow F)}^{(s)}-p$ be any one of the components of $\mathscr{A}^{(s)}-p$.

Let $x^{\prime} \in E^{\prime}, y \in F$, then $x^{\prime} \otimes y$ is a rank one operator. So $s_{n}\left(x^{\prime} \otimes y\right)=0, \forall n \geq 2$. Therefore,

$$
\beta_{A, p}^{(s)}\left(x^{\prime} \otimes y\right)=\left(\sum_{n=1}^{\infty}\left(\left|a_{n 1} s_{1}\left(x^{\prime} \otimes y\right)\right|\right)^{p}\right)^{\frac{1}{p}}=\left\|x^{\prime} \otimes y\right\|\left(\sum_{n=1}^{\infty}\left|a_{n 1}\right|^{p}\right)^{\frac{1}{p}} .
$$

Again $\left\|x^{\prime} \otimes y\right\|=\sup _{\|x\|=1}\left\|\left(x^{\prime} \otimes y\right)(x)\right\|=\left(\sup _{\|x\|=1}\left|x^{\prime}(x)\right|\right)\|y\|=\left\|x^{\prime}\right\|\|y\|$.

Therefore

$$
\hat{\beta}_{A, p}^{(s)}\left(x^{\prime} \otimes y\right)=\left\|x^{\prime}\right\|\|y\| .
$$

Suppose that $S, T \in \mathscr{A}_{(E \rightarrow F)}^{(s)}-p$, then

$$
\beta_{A, p}^{(s)}(S+T)=\left(\sum_{n=1}^{\infty}\left(\sum_{k=1}^{\infty}\left|a_{n k} s_{k}(S+T)\right|\right)^{p}\right)^{\frac{1}{p}}
$$




$$
\begin{aligned}
& =\left(\sum_{n=1}^{\infty}\left(\sum_{k=1}^{\infty}\left|a_{n, 2 k-1} s_{2 k-1}(S+T)\right|+\sum_{k=1}^{\infty}\left|a_{n, 2 k} s_{2 k}(S+T)\right|\right)^{p}\right)^{\frac{1}{p}} \\
& \leq C_{\beta} \cdot M\left(\left(\sum_{n=1}^{\infty}\left(\sum_{k=1}^{\infty}\left|a_{n k} s_{k}(S)\right|\right)^{p}\right)^{\frac{1}{p}}+\left(\sum_{n=1}^{\infty}\left(\sum_{k=1}^{\infty}\left|a_{n k} s_{k}(T)\right|\right)^{p}\right)^{\frac{1}{p}}\right) \\
& \leq C_{\beta} \cdot M\left(\beta_{A, p}^{(s)}(S)+\beta_{A, p}^{(s)}(T)\right),
\end{aligned}
$$

where $C_{\beta} \geq 1$ is a constant. Thus

$$
\hat{\beta}_{A, p}^{(s)}(S+T) \leq C_{\beta} \cdot M\left(\hat{\beta}_{A, p}^{(s)}(S)+\hat{\beta}_{A, p}^{(s)}(T)\right) .
$$

Finally, let $S \in \mathscr{A}_{(E \rightarrow F)}^{(s)}-p, R \in \mathscr{L}\left(F, F_{0}\right)$ and $T \in \mathscr{L}\left(E_{0}, E\right)$. Then

$$
\begin{aligned}
\beta_{A, p}^{(s)}(R S T) & =\left(\sum_{n=1}^{\infty}\left(\sum_{k=1}^{\infty}\left|a_{n k} s_{k}(R S T)\right|\right)^{p}\right)^{\frac{1}{p}} \\
& \leq\|R\|\|T\|\left(\left(\sum_{n=1}^{\infty}\left(\sum_{k=1}^{\infty}\left|a_{n k} s_{k}(S)\right|\right)^{p}\right)^{\frac{1}{p}}\right) \\
& \leq\|R\| \beta_{A, p}^{(s)}(S)\|T\| .
\end{aligned}
$$

Thus

$$
\hat{\beta}_{A, p}^{(s)}(R S T) \leq\|R\| \hat{\beta}_{A, p}^{(s)}(S)\|T\| .
$$

Hence $\hat{\beta}_{A, p}^{(s)}$ is a quasi-norm on the operator ideal $\mathscr{A}^{(s)}-p$.

Example 3.1. Let $A=\left(a_{n k}\right)$ be a Cesàro matrix of order 1 , then for $p=2, \hat{\beta}_{A, 2}^{(s)}$ is a quasi norm on the operator ideal $\mathscr{A}^{(s)}-2$, where $\hat{\beta}_{A, 2}^{(s)}(T)=\frac{\sqrt{6}}{\pi}\left(\sum_{n=1}^{\infty}\left(\frac{1}{n} \sum_{k=1}^{n} s_{k}(T)\right)^{2}\right)^{\frac{1}{2}}, T \in \mathscr{A}^{(s)}-2$.

Corollary 3.2. Let $A=\left(a_{n k}\right)$ be a nonzero matrix satisfying (3.1) and $\sup _{n \geq 1}\left|a_{n 1}\right|<\infty$. Then for $p=\infty$, the function $\hat{\beta}_{A, \infty}^{(s)}$ is a quasi-norm on the operator ideal $\mathscr{A}^{(s)}-\infty$, where $\hat{\beta}_{A, \infty}^{(s)}(T)=$ $\frac{\beta_{A, \infty}^{(s)}(T)}{\sup _{n \geq 1}\left|a_{n 1}\right|}, T \in \mathscr{A}^{(s)}-\infty$.

Theorem 3.3. The operator ideal $\mathscr{A}^{(s)}-p$ is complete with the quasi-norm $\hat{\beta}_{A, p}^{(s)}$, i.e., $\left[\mathscr{A}^{(s)}-p\right.$, $\hat{\beta}_{A, p}^{(s)}$ ] is a quasi-Banach operator ideal for $0<p \leq \infty$.

Proof. Let $0<p<\infty$. To prove $\mathscr{A}^{(s)}-p$ is a quasi-Banach operator ideal, it is enough to prove that each component $\mathscr{A}_{(E \rightarrow F)}^{(s)}-p$ of $\mathscr{A}^{(s)}-p$ is complete under the quasi norm $\hat{\beta}_{A, p}^{(s)}$. We have

$$
\beta_{A, p}^{(s)}(T)=\left(\sum_{n=1}^{\infty}\left(\sum_{k=1}^{\infty}\left|a_{n k} s_{k}(T)\right|\right)^{p}\right)^{\frac{1}{p}}
$$




$$
\begin{gathered}
\geq\left(\sum_{n=1}^{\infty}\left(\left|a_{n 1} s_{1}(T)\right|\right)^{p}\right)^{\frac{1}{p}} \\
=\|T\|\left(\sum_{n=1}^{\infty}\left|a_{n 1}\right|^{p}\right)^{\frac{1}{p}} . \\
\Rightarrow\|T\| \leq \hat{\beta}_{A, p}^{(s)}(T) \quad \text { for } T \in \mathscr{A}_{(E \rightarrow F)}^{(s)}-p .
\end{gathered}
$$

Let $\left(T_{m}\right)$ be a Cauchy sequence in $\mathscr{A}_{(E \rightarrow F)}^{(s)}-p$. Then $\forall \epsilon>0$, there exist $N \in \mathbb{N}$ such that

$$
\hat{\beta}_{A, p}^{(s)}\left(T_{m}-T_{l}\right)<\epsilon, \quad \forall m, l \geq N .
$$

Now from (3.4),

$$
\left\|T_{m}-T_{l}\right\| \leq \hat{\beta}_{A, p}^{(s)}\left(T_{m}-T_{l}\right)
$$

Using (3.5), we have

$$
\left\|T_{m}-T_{l}\right\| \leq \hat{\beta}_{A, p}^{(s)}\left(T_{m}-T_{l}\right)<\epsilon \quad \forall m, l \geq N .
$$

Hence $\left(T_{m}\right)$ is a Cauchy sequence in $\mathscr{L}(E, F)$. As $F$ is a Banach space, $\mathscr{L}(E, F)$ is also a Banach space. Therefore $T_{m} \rightarrow T$ as $m \rightarrow \infty$ in $\mathscr{L}(E, F)$. We shall now show that $T_{m} \rightarrow T$ as $m \rightarrow \infty$ in $\mathscr{A}_{(E \rightarrow F)}^{(s)}-p$.

Using Lemma 2.1., we have

$$
\left|s_{n}\left(T_{l}-T_{m}\right)-s_{n}\left(T-T_{m}\right)\right| \leq\left\|T_{l}-T\right\| .
$$

Letting $l \rightarrow \infty$, we have

$$
s_{n}\left(T_{l}-T_{m}\right) \rightarrow s_{n}\left(T-T_{m}\right)
$$

From (3.5), we get

$$
\left(\sum_{n=1}^{\infty}\left(\sum_{k=1}^{\infty}\left|a_{n k} s_{k}\left(T_{l}-T_{m}\right)\right|\right)^{p}\right)^{\frac{1}{p}}<\epsilon\left(\sum_{n=1}^{\infty}\left|a_{n 1}\right|^{p}\right)^{\frac{1}{p}}, \quad \forall m, l \geq N .
$$

Using (3.6), it can be shown that as $l \rightarrow \infty$ ( keeping $m \geq N$ fixed)

$$
\begin{aligned}
& \left(\sum_{n=1}^{\infty}\left(\sum_{k=1}^{\infty}\left|a_{n k} s_{k}\left(T-T_{m}\right)\right|\right)^{p}\right)^{\frac{1}{p}} \leq \epsilon\left(\sum_{n=1}^{\infty}\left|a_{n 1}\right|^{p}\right)^{\frac{1}{p}} \\
\Rightarrow & \hat{\beta}_{A, p}^{(s)}\left(T-T_{m}\right) \leq \epsilon \quad \forall m \geq N .
\end{aligned}
$$

This means that $T_{m} \rightarrow T$ under the quasi-norm $\hat{\beta}_{A, p}^{(s)}$. Next to show that $T \in \mathscr{A}_{(E \rightarrow F)}^{(s)}-p$. Now

$$
\sum_{k=1}^{\infty}\left|a_{n k} s_{k}(T)\right|=\sum_{k=1}^{\infty}\left|a_{n, 2 k-1} s_{2 k-1}(T)\right|+\sum_{k=1}^{\infty}\left|a_{n, 2 k} s_{2 k}(T)\right|
$$




$$
\leq \sum_{k=1}^{\infty}\left(\left|a_{n, 2 k-1}\right|+\left|a_{n, 2 k}\right|\right) s_{2 k-1}(T)
$$

Since $0 \leq s_{n+1}(T) \leq s_{n}(T), \forall n$ and using the inequality (3.1), we have

$$
\sum_{k=1}^{\infty}\left|a_{n k} s_{k}(T)\right| \leq M\left(\sum_{k=1}^{\infty}\left|a_{n k}\right| s_{k}\left(T-T_{N}\right)+\sum_{k=1}^{\infty}\left|a_{n k}\right| s_{k}\left(T_{N}\right)\right) .
$$

Therefore

$$
\begin{aligned}
\left(\sum_{n=1}^{\infty}\left(\sum_{k=1}^{\infty}\left|a_{n k} s_{k}(T)\right|\right)^{p}\right)^{\frac{1}{p}} & \leq C \cdot M\left[\left(\sum_{n=1}^{\infty}\left(\sum_{k=1}^{\infty}\left|a_{n k} s_{k}\left(T-T_{N}\right)\right|\right)^{p}\right)^{\frac{1}{p}}+\left(\sum_{n=1}^{\infty}\left(\sum_{k=1}^{\infty}\left|a_{n k} s_{k}\left(T_{N}\right)\right|\right)^{p}\right)^{\frac{1}{p}}\right] \\
& <\infty,
\end{aligned}
$$

since $\hat{\beta}_{A, p}^{(s)}\left(T-T_{N}\right)<\infty$ and $\left(T_{N}\right) \in \mathscr{A}_{(E \rightarrow F)}^{(s)}-p$.

Hence $T \in \mathscr{A}_{(E \rightarrow F)}^{(s)}-p$.

For $p=\infty$, we can similarly prove that $\mathscr{A}^{(s)}-\infty$ is complete under the quasi-norm $\hat{\beta}_{A, p}^{(s)}-$ $\infty$.

This completes the proof.

We now study some properties of the quasi-Banach operator ideal $\mathscr{A}^{(s)}-p$ for $0<p \leq \infty$.

Theorem 3.4. If the $s$-number sequence is injective, then the quasi-Banach operator ideal $\left[\mathscr{A}^{(s)}-p, \hat{\beta}_{A, p}^{(s)}\right]$ is injective for $0<p \leq \infty$.

Proof. Let $0<p<\infty$. Let $T \in \mathscr{L}(E, F)$ and $J \in \mathscr{L}\left(F, F_{0}\right)$ be any metric injection. Suppose that $J T \in \mathscr{A}_{\left(E \rightarrow F_{0}\right)}^{(s)}-p$. Then

$$
\sum_{n=1}^{\infty}\left(\sum_{k=1}^{\infty}\left|a_{n k} s_{k}(J T)\right|\right)^{p}<\infty .
$$

Since the $s$-number sequence $s=\left(s_{n}\right)$ is injective, we have $s_{n}(T)=s_{n}(J T)$, for all $T \in \mathscr{L}(E, F)$, $n=1,2, \cdots$. Hence

$$
\sum_{n=1}^{\infty}\left(\sum_{k=1}^{\infty}\left|a_{n k} s_{k}(T)\right|\right)^{p}=\sum_{n=1}^{\infty}\left(\sum_{k=1}^{\infty}\left|a_{n k} s_{k}(J T)\right|\right)^{p}<\infty .
$$

Thus $T \in \mathscr{A}_{(E \rightarrow F)}^{(s)}-p$ and clearly $\hat{\beta}_{A, p}^{(s)}(J T)=\hat{\beta}_{A, p}^{(s)}(T)$ holds.

Similarly we can prove for $p=\infty$. Hence the operator ideal $\left[\mathscr{A}^{(s)}-p, \hat{\beta}_{A, p}^{(s)}\right]$ is injective.

Remark 3.3. The quasi-Banach operator ideal $\left[\mathscr{A}^{(c)}-p, \hat{\beta}_{A, p}^{(c)}\right]$ formed by Gel'fand numbers $c=$ $\left(c_{n}\right)$ and the quasi-Banach operator ideal $\left[\mathscr{A}^{(x)}-p, \hat{\beta}_{A, p}^{(x)}\right]$ formed by Weyl numbers $x=\left(x_{n}\right)$ are injective quasi-Banach operator ideals for $0<p \leq \infty$.

Theorem 3.5. If the $s$-number sequence is surjective, then the quasi-Banach operator ideal $\left[\mathscr{A}^{(s)}-p, \hat{\beta}_{A, p}^{(s)}\right]$ is surjective for $0<p \leq \infty$. 
Proof. Let $0<p<\infty$. Let $T \in \mathscr{L}(E, F)$ and $Q \in \mathscr{L}\left(E_{0}, E\right)$ be any metric surjection. Suppose that $T Q \in \mathscr{A}_{\left(E_{0} \rightarrow F\right)}^{(s)}-p$. Then

$$
\sum_{n=1}^{\infty}\left(\sum_{k=1}^{\infty}\left|a_{n k} s_{k}(T Q)\right|\right)^{p}<\infty .
$$

Since the $s$-number sequence $s=\left(s_{n}\right)$ is surjective, we have $s_{n}(T)=s_{n}(T Q)$, for all $T \in \mathscr{L}(E, F)$ and $n=1,2, \cdots$. Hence

$$
\sum_{n=1}^{\infty}\left(\sum_{k=1}^{\infty}\left|a_{n k} s_{k}(T)\right|\right)^{p}=\sum_{n=1}^{\infty}\left(\sum_{k=1}^{\infty}\left|a_{n k} s_{k}(T Q)\right|\right)^{p}<\infty .
$$

Thus $T \in \mathscr{A}_{(E \rightarrow F)}^{(s)}-p$ and also $\hat{\beta}_{A, p}^{(s)}(T Q)=\hat{\beta}_{A, p}^{(s)}(T)$.

It is easy to check for $p=\infty$. Hence the operator ideal $\left[\mathscr{A}^{(s)}-p, \hat{\beta}_{A, p}^{(s)}\right.$ ] is surjective.

Remark 3.4. The quasi-Banach operator ideal $\left[\mathscr{A}^{(d)}-p, \hat{\beta}_{A, p}^{(d)}\right]$ formed by Kolmogorov numbers $d=\left(d_{n}\right)$ and the quasi-Banach operator ideal $\left[\mathscr{A}^{(y)}-p, \hat{\beta}_{A, p}^{(y)}\right]$ formed by Chang numbers $y=\left(y_{n}\right)$ are surjective quasi-Banach operator ideals.

Let us consider $\left[\mathscr{A}^{(a)}-p, \hat{\beta}_{A, p}^{(a)}\right]$ and $\left[\mathscr{A}^{(h)}-p, \hat{\beta}_{A, p}^{(h)}\right]$ be the quasi-Banach operator ideals corresponding to the approximation numbers $a=\left(a_{n}\right)$ and the Hilbert numbers $h=\left(h_{n}\right)$ respectively. Then we have the following inclusion relations among the operator ideals.

Theorem 3.6. Let $0<p \leq \infty$. Then

(I) $\mathscr{A}^{(a)}-p \subseteq \mathscr{A}^{(c)}-p \subseteq \mathscr{A}^{(x)}-p \subseteq \mathscr{A}^{(h)}-p \quad$ and

(II) $\mathscr{A}^{(a)}-p \subseteq \mathscr{A}^{(d)}-p \subseteq \mathscr{A}^{(y)}-p \subseteq \mathscr{A}^{(h)}-p$.

Proof. Let $0<p<\infty$. Suppose that $T \in \mathscr{A}^{(a)}-p$. Then

$$
\sum_{n=1}^{\infty}\left(\sum_{k=1}^{\infty}\left|a_{n k} a_{k}(T)\right|\right)^{p}<\infty
$$

From Proposition 2.1., we have

$$
\sum_{n=1}^{\infty}\left(\sum_{k=1}^{\infty}\left|a_{n k} h_{k}(T)\right|\right)^{p} \leq \sum_{n=1}^{\infty}\left(\sum_{k=1}^{\infty}\left|a_{n k} x_{k}(T)\right|\right)^{p} \leq \sum_{n=1}^{\infty}\left(\sum_{k=1}^{\infty}\left|a_{n k} c_{k}(T)\right|\right)^{p} \leq \sum_{n=1}^{\infty}\left(\sum_{k=1}^{\infty}\left|a_{n k} a_{k}(T)\right|\right)^{p} .
$$

Hence the proof of $(I)$ follows for $0<p<\infty$. It is trivial to check for $p=\infty$.

We omit the proof of $(I I)$ as it is similar to the previous one.

There are some converse estimates among $s$-number sequences as given below.

Lemma 3.2 ([8], p.165). Let $T \in \mathscr{L}(E, F)$. Then $a_{n}(T) \leq 2 n^{\frac{1}{2}} c_{n}(T)$ and $a_{n}(T) \leq 2 n^{\frac{1}{2}} d_{n}(T)$.

We have next result related to this converse estimates. 
Theorem 3.7. Let $0<r, p<\infty$ and $A=\left(a_{n k}\right)$ be a diagonal matrix where

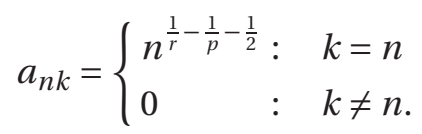

If a bounded linear operator $T$ from $E$ to $F$ belongs to $\mathscr{L}_{r, p}^{(c)}$, then $T$ belongs to $\mathscr{A}^{(a)}-p$.

Proof. For $0<p<\infty$, we have

$$
\begin{aligned}
\sum_{n=1}^{\infty}\left(\sum_{k=1}^{\infty}\left|a_{n k} a_{k}(T)\right|\right)^{p} & =\sum_{n=1}^{\infty}\left(n^{\frac{1}{r}-\frac{1}{p}-\frac{1}{2}} a_{n}(T)\right)^{p} \\
& \leq \sum_{n=1}^{\infty}\left(n^{\frac{1}{r}-\frac{1}{p}-\frac{1}{2}} \cdot 2 n^{\frac{1}{2}} c_{n}(T)\right)^{p} \\
& =2^{p} \sum_{n=1}^{\infty}\left(n^{\frac{1}{r}-\frac{1}{p}} c_{n}(T)\right)^{p}<\infty .
\end{aligned}
$$

(Using Lemma 3.1.)

Hence the result follows.

Remark 3.5. In particular, if we take the diagonal matrix $A=\left(a_{n k}\right)$, where

$$
a_{n k}= \begin{cases}n^{-\frac{1}{2}}: & k=n \\ 0: & k \neq n .\end{cases}
$$

If a bounded linear operator $T$ from $E$ to $F$ belongs to $S_{p}^{(c)}$, then $T$ belongs to $\mathscr{A}^{(a)}-p$ for $0<p<\infty$.

Theorem 3.8. Let $0<r, p<\infty$ and $A=\left(a_{n k}\right)$ be a diagonal matrix where

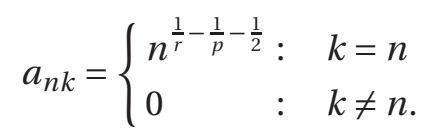

If a bounded linear operator $T$ from $E$ to $F$ belongs to $\mathscr{L}_{r, p}^{(d)}$, then $T$ belongs to $\mathscr{A}^{(a)}-p$.

Proof. The proof is similar to the proof of Theorem 3.7.

Remark 3.6. In particular, if we take the diagonal matrix $A=\left(a_{n k}\right)$, where

$$
a_{n k}= \begin{cases}n^{-\frac{1}{2}}: & k=n \\ 0: & k \neq n .\end{cases}
$$

If a bounded linear operator $T$ from $E$ to $F$ belongs to $S_{p}^{(d)}$, then $T$ belongs to $\mathscr{A}^{(a)}-p$ for $0<$ $p<\infty$.

We now state the dual of the operator ideal formed by different $s$-number sequences. 
Theorem 3.9. The operator ideal $\mathscr{A}^{(a)}-p$ is symmetric and the operator ideal $\mathscr{A}^{(h)}-p$ is completely symmetric for $0<p \leq \infty$.

Proof. Since $a_{n}\left(T^{\prime}\right) \leq a_{n}(T)$ and $h_{n}\left(T^{\prime}\right)=h_{n}(T)$, for all $T \in \mathscr{L}(E, F)$, we have $\mathscr{A}^{(a)}-p \subseteq$ $\left(\mathscr{A}^{(a)}-p\right)^{\prime}$ and $\mathscr{A}^{(h)}-p=\left(\mathscr{A}^{(h)}-p\right)^{\prime}$.

Theorem 3.10. Let $0<p \leq \infty$. Then $\mathscr{A}^{(c)}-p=\left(\mathscr{A}^{(d)}-p\right)^{\prime}$ and $\mathscr{A}^{(d)}-p \subseteq\left(\mathscr{A}^{(c)}-p\right)^{\prime}$. In addition, if $T$ belongs to the class of compact operators, then $\mathscr{A}^{(d)}-p=\left(\mathscr{A}^{(c)}-p\right)^{\prime}$.

Proof. The proof follows from Theorem 2.2.

Theorem 3.11. Let $0<p \leq \infty$. Then $\mathscr{A}^{(x)}-p=\left(\mathscr{A}^{(y)}-p\right)^{\prime}$ and $\mathscr{A}^{(y)}-p=\left(\mathscr{A}^{(x)}-p\right)^{\prime}$.

Proof. The proof follows from Theorem 2.3.

\subsection{Small operator ideal}

This section deals with the small ideals of operators. In [6], Pietsch proved that the ideal $S_{p}^{(a)}$ is small for $0<p<\infty$. Here, we proved that the ideal formed by approximation type ces $_{p}$ operators is small for $1<p<\infty$.

Let $A$ be a Cesàro matrix of order 1 and $\mathscr{A}^{(a)}-p$ be an ideal of approximation type ces $_{p}$ operators.

Then we have the following theorem.

Theorem 3.12. The quasi-Banach operator ideal $\mathscr{A}^{(a)}-p$ of approximation type ces opera- $^{(a)}$ tors is small for $1<p<\infty$.

Proof. Let $\lambda=\left(\sum_{n=1}^{\infty} \frac{1}{n^{p}}\right)^{\frac{1}{p}}$ for $1<p<\infty$. Then $\left[\mathscr{A}^{(a)}-p, \hat{\beta}_{A, p}^{(a)}\right]$ is a quasi-Banach operator ideal, where $\hat{\beta}_{A, p}^{(a)}(T)=\frac{1}{\lambda}\left(\sum_{n=1}^{\infty}\left(\frac{1}{n} \sum_{k=1}^{n} a_{k}(T)\right)^{p}\right)^{\frac{1}{p}}$. Let $E, F$ be any two Banach spaces. Suppose that $\mathscr{A}_{(E \rightarrow F)}^{(a)}-p=\mathscr{L}(E, F)$, then there exists a constant $C>0$ such that $\hat{\beta}_{A, p}^{(a)}(T) \leq C\|T\|$ for all $T \in \mathscr{L}(E, F)$. Assume that $E$ and $F$ both are infinite dimensional Banach spaces. Then by Dvoretzky's theorem [7] for $m=1,2, \cdots$ we have quotient spaces $E / N_{m}$ and subspaces $M_{m}$ of $F$ which can be mapped onto $l_{2}^{m}$ by isomorphisms $X_{m}$ and $A_{m}$ such that $\left\|X_{m}\right\|\left\|X_{m}^{-1}\right\| \leq 2$ and $\left\|A_{m}\right\|\left\|A_{m}^{-1}\right\| \leq 2$. Consider $I_{m}$ be the identity map on $l_{2}^{m}, Q_{m}$ be the quotient map from $E$ onto $E / N_{m}$ and $J_{m}$ be the natural embedding map from $M_{m}$ into $F$. Let $a_{n}, d_{n}$ and $u_{n}$ be approximation numbers, Kolmogorov numbers and Bernstein numbers [5], respectively. Then

$$
1=u_{n}\left(I_{m}\right)=u_{n}\left(A_{m} A_{m}^{-1} I_{m} X_{m} X_{m}^{-1}\right)
$$




$$
\begin{aligned}
& \leq\left\|A_{m}\right\| u_{n}\left(A_{m}^{-1} I_{m} X_{m}\right)\left\|X_{m}^{-1}\right\| \\
& =\left\|A_{m}\right\| u_{n}\left(J_{m} A_{m}^{-1} I_{m} X_{m}\right)\left\|X_{m}^{-1}\right\| \\
& \leq\left\|A_{m}\right\| d_{n}\left(J_{m} A_{m}^{-1} I_{m} X_{m}\right)\left\|X_{m}^{-1}\right\| \\
& =\left\|A_{m}\right\| d_{n}\left(J_{m} A_{m}^{-1} I_{m} X_{m} Q_{m}\right)\left\|X_{m}^{-1}\right\| \\
& \leq\left\|A_{m}\right\| a_{n}\left(J_{m} A_{m}^{-1} I_{m} X_{m} Q_{m}\right)\left\|X_{m}^{-1}\right\| \quad \text { for } n=1,2, \cdots, m .
\end{aligned}
$$

Now

$$
\begin{aligned}
\sum_{k=1}^{n}(1) & \leq \sum_{k=1}^{n}\left\|A_{m}\right\| a_{k}\left(J_{m} A_{m}^{-1} I_{m} X_{m} Q_{m}\right)\left\|X_{m}^{-1}\right\| \\
\Rightarrow \frac{1}{n} \cdot n & \leq\left\|A_{m}\right\|\left(\frac{1}{n} \sum_{k=1}^{n} a_{k}\left(J_{m} A_{m}^{-1} I_{m} X_{m} Q_{m}\right)\right)\left\|X_{m}^{-1}\right\| \\
\Rightarrow 1 & \leq\left(\left\|A_{m}\right\|\left\|X_{m}^{-1}\right\|\right)^{p}\left(\frac{1}{n} \sum_{k=1}^{n} a_{k}\left(J_{m} A_{m}^{-1} I_{m} X_{m} Q_{m}\right)\right)^{p} .
\end{aligned}
$$

Therefore

$$
\begin{aligned}
\left(\sum_{n=1}^{m}(1)\right)^{\frac{1}{p}} & \leq\left(\left\|A_{m}\right\|\left\|X_{m}^{-1}\right\|\right)\left(\sum_{n=1}^{m}\left(\frac{1}{n} \sum_{k=1}^{n} a_{k}\left(J_{m} A_{m}^{-1} I_{m} X_{m} Q_{m}\right)\right)^{p}\right)^{\frac{1}{p}} \\
\Rightarrow \frac{1}{\lambda} m^{\frac{1}{p}} & \leq\left\|A_{m}\right\|\left\|X_{m}^{-1}\right\| \frac{1}{\lambda}\left(\sum_{n=1}^{m}\left(\frac{1}{n} \sum_{k=1}^{n} a_{k}\left(J_{m} A_{m}^{-1} I_{m} X_{m} Q_{m}\right)\right)^{p}\right)^{\frac{1}{p}} \\
\Rightarrow \frac{1}{\lambda} m^{\frac{1}{p}} & \leq\left\|A_{m}\right\|\left\|X_{m}^{-1}\right\| \hat{\beta}_{A, p}^{(a)}\left(J_{m} A_{m}^{-1} I_{m} X_{m} Q_{m}\right) \\
& \leq C\left\|A_{m}\right\|\left\|X_{m}^{-1}\right\|\left\|J_{m} A_{m}^{-1} I_{m} X_{m} Q_{m}\right\| \\
& \leq C\left\|A_{m}\right\|\left\|X_{m}^{-1}\right\|\left\|J_{m} A_{m}^{-1}\right\|\left\|I_{m}\right\|\left\|X_{m} Q_{m}\right\| \\
& =C\left\|A_{m}\right\|\left\|X_{m}^{-1}\right\|\left\|A_{m}^{-1}\right\|\left\|X_{m}\right\| \\
& \leq 4 C .
\end{aligned}
$$

This is a contradiction as $m$ is any arbitrary number. Thus $E$ and $F$ both cannot be infinite dimensional when $\mathscr{A}_{(E \rightarrow F)}^{(a)}-p=\mathscr{L}(E, F)$. This completes the proof.

Theorem 3.13. The quasi-Banach operator ideal $\mathscr{A}^{(d)}-p$ of Kolmogorov type ces $s_{p}$ operators is small for $1<p<\infty$.

Proof. The proof is similar to the proof of Theorem 3.12.

\section{Acknowledgment}

The authors are thankful to the referee for his/her valuable comments and suggestions which improved the version of the paper. This work was supported by CSIR, New Delhi, Grant 09/081(1120)/2011-EMR-I. 


\section{References}

[1] B. Carl and A. Hinrichs, On s-numbers and Weyl inequalities of operators in Banach spaces, Bull. Lond. Math. Soc., 41 (2009), 332-340.

[2] Gh. Constantin, Operators of ces - p type, Rend. Acc. Naz. Lincei., 52 (1972), 875-878.

[3] C. V. Hutton, On the approximation numbers of an operator and its adjoint, Math. Ann., 210(1974), 277-280.

[4] A. Pietsch, Einige neue klassen von kompakten linearen Abbildungen, Rev. Math. Pures Appl. (Bucarest), 8 (1963), 427-447.

[5] A. Pietsch, s-numbers of operators in Banach spaces, Studia Math. 51 (1974), 201-223.

[6] A. Pietsch, Small ideals of operators, Studia Math., 51 (1974), 265-267.

[7] A. Pietsch, Operator Ideals, VEB Deutscher Verlag der Wissenschaften, Berlin, 1978.

[8] A. Pietsch, Weyl numbers and eigenvalues of operators in Banach spaces, Math. Ann., 247 (1980), 149-168.

[9] A. Pietsch, Eigenvalues and s-numbers, Cambridge University Press, New York, NY, USA, 1986.

[10] J. R. Retherford, Applications of Banach ideals of operators, Bull. Amer. Math. Soc., 81 (1975), 978-1012.

[11] B. E. Rhoades, Operators of $A-p$ type, Atti Accad. Naz. Lincei Rend. Cl. Sci. Fis. Mat. Natur. (8), 59 (1975), no.3-4, 238-241 (1976).

[12] B. E. Rhoades, Some sequence spaces which include the $l^{p}$ spaces, Tamkang J. Math., 10 (1979), 263-267.

[13] J. S. Shiue, On the Cesàro sequence spaces, Tamkang J. Math. , 1 (1970), 19-25.

[14] C. S. Wang, On Nörlund sequence spaces, Tamkang J. Math., 9 (1978), 269-274.

Department of Mathematics, Indian Institute of Technology Kharagpur, Kharagpur 721 302, West Bengal, India.

E-mail: amit.iitm07@gmail.com

Department of Mathematics, Indian Institute of Technology Kharagpur, Kharagpur 721 302, West Bengal, India.

E-mail: pds@maths.iitkgp.ernet.in 